\title{
Effect of COVID-19 on Mental Health Rehabilitation Centers
}

\author{
Alifiya Aamir • Sana Awan · Renato de Filippis $(\mathbb{D} \cdot$ Mufaddal Najmuddin Diwan • \\ Irfan Ullah (iD
}

Received: 4 August 2020/Accepted: 3 October 2020/Published online: 21 October 2020

(C) Springer Nature India Private Limited 2020

\begin{abstract}
The SARS-CoV-2 outbreak is an unprecedented event in modern history worldwide. To facilitate speedy and smooth recovery during this time period, an added responsibility is placed upon rehabilitation center counselors, staff and management. In this paper we expose the role that psychiatric rehabilitation plays during the COVID-19 crisis. Since
\end{abstract}

\footnotetext{
A. Aamir · S. Awan · M. N. Diwan

Department of Internal Medicine, Dow University of

Health Sciences, Karachi, Pakistan

e-mail: alifiya.aamir521@gmail.com

S. Awan

e-mail: sana.awan527@gmail.com

M. N. Diwan

e-mail: mufdiwan@gmail.com

R. de Filippis $(\square)$

Department of Health Sciences, Psychiatric Unit,

University Magna Graecia of Catanzaro, Viale Europa,

88100 Catanzaro, Italy

e-mail: defilippisrenato@gmail.com

I. Ullah

Kabir Medical College, Gandhara University, Peshawar,

Pakistan

e-mail: irfanullahecp2@gmail.com

I. Ullah

Undergraduate Research Organization, Dhaka,

Bangladesh

I. Ullah

Naseer Teaching Hospital, Peshawar, Pakistan
}

COVID-19 pandemic has remarkably raised mental health concerns, one of the high risks and possibly neglected groups includes individuals undergoing mental health rehabilitation, the impact on which can be significant as compared to the rest of the population. In order to ensure that individuals on the road to recovery are not alone and forgotten during the times of coronavirus crisis they should be provided with adequate resources, such as online meetings and group therapy sessions, assessment, treatment and support by telephone and video consultation and getting the medications they need to recover. This article highlights how the coronavirus pandemic, that has hit hard the health care and all categories of patients affected by mental disorders, is likely to be particularly dramatic for mental health rehabilitation centers. Indeed, the COVID-19 crisis has severely tested the health systems worldwide, reducing sometimes the quality and quantity of care offered to psychiatric patients both hospitalized and not in rehabilitation centers. The main goal of this paper is to raise awareness of the importance, often underestimated, of mental health rehabilitation centers, on which one should invest for the both future of psychological and psychiatric rehabilitation and the current crisis as well.

Keywords COVID-19 - Coronavirus - Mental health $\cdot$ Rehabilitation $\cdot$ Rehabilitation centers 
Severe acute respiratory syndrome coronavirus 2 (SARS-CoV-2) responsible for coronavirus disease 2019 (COVID-19), which began as a few cases of a flu like disease in Wuhan, China, is now a pandemic that has affected millions of people globally and has forced upon unprecedented changes in every field of life [1]. During these times of crisis, inevitably people are expected to develop fear of the unknown and resist change, the magnitude of which has brought about population-wide psychological impact. Due to the ongoing pandemic, individuals are most likely to develop feelings of anxiety and uncertainty due to fear of contracting the virus, whereas with all the changes and adjustments to daily routines, it has also brought on financial pressures, social isolation and unhealthy habits, therefore the impact of which on individuals undergoing rehabilitation is likely to be significant [2]. During a previous influenza A H1N1v outbreak in the UK in 2009 , around $10 \%$ to $30 \%$ of the general public were very or fairly worried about the possibility of contracting the virus [2]. Consequently, there has been a striking increase in cases of depression, anxiety, and substance abuse [2]. Indeed, recent researches suggest that addiction problems are likely to worsen during the lockdown instead of slowing down [3]. Importantly, the COVID-19 affected patients particularly those in isolation are likely to express stress, anxiety and depressive symptoms. Such behavior has previously been reported from SARS and MERS cases, which found that many of those patients experienced some degree of confusion (27.9\%, 36 of 129 patients), depressed mood (32.6\%, 42 of 129), anxiety (35.7\%, 46 of 129), impaired memory (34.1\%, 44 of 129), and insomnia (41.9\%, 54 of 129) [4]. In a meta-analysis, the point prevalence in the post-illness stage of these cases was found to be $32.2 \%$ (95\% CI 23.7-42.0\%) for post-traumatic stress disorder (PTSD), $14.9 \%$ $(12.1-18.2 \%)$ for depression, and $14.8 \%$ (11.1-19.4\%) for anxiety [5].

Since COVID-19 pandemic has remarkably raised mental health concerns, one of the high risks and possibly neglected groups includes individuals undergoing mental health rehabilitation, the impact on which can be significant as compared to the rest of the population. This can be due to pre-existing depression and anxiety disorders in such individuals that can exacerbate in the times of crisis and shake the foundations of stability or recovery for them. To facilitate speedy and smooth recovery of such individuals during this time period, an added responsibility is placed upon rehabilitation center counselors, staff and management [6].

As social distancing is considered to be the key preventive measure for infection control, mental health rehabilitation day boarding and day care centers, being a non-emergency service have limited new admissions, cancelled programs and have been temporarily closed at some places which in turn is going to significantly deprive people with chronic mental illnesses and intellectual disabilities of these services [7]. The clinical outcome for these individuals will be adverse with a risk of aggravation of symptoms and even a relapse. Therefore, it is imperative to develop home-based rehabilitation strategies such as telepsychiatry in order to continue therapy for them [8].

Individuals with pre-existing mental health problems are generally at high risk of infections, hence they are inevitably at an increased risk of COVID-19 [9]. Some of them are of older age and on medications, and being identified as a vulnerable group can contribute to heightened anxiety [10]. For patients with psychiatric disorders, it is expected of them to exhibit irrational behavior as many have been reported to show increased stress about their physical health, anger, impulsivity, and suicidal ideation [11]. It may be difficult for people with schizophrenia and other related disorders to practice and adhere to the protective measures recommended to prevent infection such as hand washing, wearing masks [12]. Of note, it is a major challenge for individuals in rehabilitation to practice social distancing, as social interaction for them is not a luxury, but rather a form of therapy [13]. Group interactions and projects are part of their treatment where they walk the grounds, dine in communal areas, watch television together in day rooms, exercise and go to therapy together. The peersupport groups meetings and activities are such a vital source of emotional and spiritual support to people struggling to stay in recovery. Hence isolation can be dangerous and the fear of contracting a life-threatening illness is unlikely to promote psychiatric healing. The psychology of fear associated with this virus can result in elevated levels of anxiety and depression which may have a negative impact on recovery [14]. One of the major challenges faced by many individuals with underlying mental disorders and in recovery is prolonged isolation, which is likely to cause anxiety 
and depression that can be damaging if they are required to stay at home until the pandemic lasts. Isolation is considered as a negative notion when it comes to mental health rehabilitation, hence forcing people to isolate will likely have adverse effects on recovery [15].

In the fight against COVID-19 pandemic, there is a need to address mental health challenges and develop strategies and interventions to ensure continued quality care. Adequate measures are being taken in some countries like the USA, such as regular testing of patients and potential patients for COVID-19, adapting their programs to comply with social-distancing guidelines such as the use of telepsychiatry to limit the exposure possibility [16]. There is a shift observed from on-site facilities and group therapies to virtual or phone conferencing platforms. These strategies will be effective to mitigate spread of COVID-19 in vulnerable communities and simultaneously provide adequate care to mental health rehab patients.

One emerging consequence of COVID-19 is the presence of post-intensive care syndrome (PICS) characterized by symptoms involving physical strength deficits, cognitive decline, and mental health disturbances observed after discharge from critical care that persist for a protracted amount of time [17]. Therefore, patients surviving intensive care units (ICU) and mechanical ventilation for several weeks and other severe conditions will require early rehabilitation and other interventions. It is imperative to establish an integrated rehabilitation response for patients recovering from critical care at hospital with the help of psychologists, physical and occupational therapists etc. This issue needs additional attention in low resource countries where resources and workforce required for rehabilitation are inadequate [18].

In order to ensure that all individuals on the road to recovery and/or admitted to a rehabilitation center are not alone and forgotten during the times of coronavirus crisis they should be provided with adequate resources, such as online meetings and group therapy sessions, assessment, treatment and support by telephone and video consultation and getting the medications they need to recover.

Author contributions All authors contributed to the article and approved the submitted version.Funding No funding was provided for this work.
Compliance with Ethical Standards

Conflict of interest Authors declare no competing interests.

\section{References}

1. Sifuentes-Rodríguez E, Palacios-Reyes D. COVID-19: the outbreak caused by a new coronavirus. Bol Med Hosp Infant Mex. 2020;77. https://www.bmhim.com/frame_esp. php?id=124

2. Wang C, Pan R, Wan X, Tan Y, Xu L, Ho CS, et al. Immediate psychological responses and associated factors during the initial stage of the 2019 coronavirus disease (COVID-19) epidemic among the general population in China. Int J Environ Res Public Health. 2020;17:1729.

3. Lapeyre-Mestre M, Boucher A, Daveluy A, Gibaja V, Jouanjus E, Mallaret M, et al. Addictovigilance contribution during COVID-19 epidemic and lockdown in France. Therapies. 2020. https://linkinghub.elsevier.com/retrieve/ pii/S0040595720301104

4. Sommer IE, Bakker PR. What can psychiatrists learn from SARS and MERS outbreaks? Lancet Psychiatry. 2020;7:565-6.

5. Rogers JP, Chesney E, Oliver D, Pollak TA, McGuire P, Fusar-Poli P, et al. Psychiatric and neuropsychiatric presentations associated with severe coronavirus infections: a systematic review and meta-analysis with comparison to the COVID-19 pandemic. Lancet Psychiatry. 2020;7:611-27.

6. Banyan Treatment Center. Coronavirus protocol for rehab. https://www.banyantreatmentcenter.com/coronavirusprotocol-for-rehab/

7. Chaturvedi SK. Covid-19, coronavirus and mental health rehabilitation at times of crisis. J Psychosoc Rehabil Ment Heal. 2020;7:1-2.

8. Schuh Teixeira AL, Spadini AV, Pereira-Sanchez V, Ojeahere MI, Morimoto K, Chang A, et al. The urge to implement and expand telepsychiatry during the COVID-19 crisis: early career psychiatrists' perspective. Rev Psiquiatr Salud Ment. 2020;13:174-5.

9. Zhu Y, Chen L, Ji H, Xi M, Fang Y, Li Y. The risk and prevention of novel coronavirus pneumonia infections among inpatients in psychiatric hospitals. Neurosci Bull. 2020;36:299-302.

10. Webb L. COVID-19 lockdown: a perfect storm for older people's mental health. J Psychiatr Ment Health Nurs. 2020;jpm.12644. https://onlinelibrary.wiley.com/doi/abs/ 10.1111/jpm.12644

11. Hao F, Tan W, Jiang L, Zhang L, Zhao X, Zou Y, et al. Do psychiatric patients experience more psychiatric symptoms during COVID-19 pandemic and lockdown? A case-control study with service and research implications for immunopsychiatry. Brain Behav Immun. 2020;87:100-6.

12. Maguire PA, Reay RE, Looi JC. Nothing to sneeze atuptake of protective measures against an influenza pandemic by people with schizophrenia: willingness and perceived barriers. Australas Psychiatry. 2019;27:171-8.

13. Whitley R, McKenzie K. Social capital and psychiatry: review of the literature. Harv Rev Psychiatry. 2005;13:71-84. 
14. Moreno C, Wykes T, Galderisi S, Nordentoft M, Crossley $\mathrm{N}$, Jones $\mathrm{N}$, et al. How mental health care should change as a consequence of the COVID-19 pandemic. Lancet Psychiatry. 2020;7:813-24.

15. Courtet P, Olié E, Debien C, Vaiva G. Keep Socially (but not physically) connected and carry on. J Clin Psychiatry. 2020;81. https://www.psychiatrist.com/JCP/article/Pages/ 2020/v81/20com13370.aspx

16. Pinals DA, Hepburn B, Parks J, Stephenson AH. The behavioral health system and its response to COVID-19: a snapshot perspective. Psychiatr Serv. 2020;71:1070-4.
17. Jaffri A, Jaffri UA. Post-intensive care syndrome and COVID-19: crisis after a crisis? Hear Lung. 2020. https:// linkinghub.elsevier.com/retrieve/pii/S0147956320302661

18. Rathore FA, Ilyas A. Post-intensive care syndrome and COVID-19: crisis after a crisis? Hear Lung Circ. 2020. https://linkinghub.elsevier.com/retrieve/pii/ S1443950620304443

Publisher's Note Springer Nature remains neutral with regard to jurisdictional claims in published maps and institutional affiliations. 